\title{
Texture Measuring by Means of Perceptually-Based Fineness Functions
}

\author{
J. Chamorro-Martínez and P. Martínez-Jiménez* \\ Department of Computer Science and Artificial Intelligence, University of Granada \\ C/ Periodista Daniel Saucedo Aranda s/n, 18071 Granada, Spain \\ \{jesus,pedromartinez\}@decsai.ugr.es
}

\begin{abstract}
Although texture is a widely analyzed property in computer vision, models which relate this feature with human perception are needed. In this paper, a model that associates computational fineness measures with the human perception of this type of texture property is proposed. The fineness perception is collected from human subjects, performing an aggregation of their assessments. Considering a wide variety of measures, a coarseness-fineness function relating these features with the human assesments is obtained as the function which provides the best fit of the collected data.
\end{abstract}

Keywords: Texture, image features, textural features, human perception, coarseness, fineness.

\section{Introduction}

Texture is, together with the color and shape, one of the most important features used in the analysis of natural images. It is usual to describe visual textures according to some properties like coarseness-fineness, orientation or regularity $[12$. From all of them, the coarseness-fineness is one of the most popular, being common to associate the presence of texture with the presence of fineness.

Different approaches for texture characterization have been developed over the years 345. Haralick 3] proposed a classification of such approaches in two main groups: statistical methods (which analyze gray tone spatial distribution by computing some statistics on pixel intensities) and structural methods (which characterize texture by means of texels arranged in a certain way that is given by a set of rules). However, one of the main drawback of theses methods is that there is not a clear perceptual relationship between the measure value and the way the humans perceive the texture.

One of the first papers where human perception of texture is taken into account was presented by Tamura 2. However, in Tamura's paper the relationship between the computational measures and the human perception of the different textural features is not learnt, just a rank correlation value is given. More recent approaches perform experiments with humans in order to model human

\footnotetext{
* This work was supported by Spanish research programme Consolider Ingenio 2010: MIPRCV (CSD2007-00018).
} 
perception, but it is usual that the results given by such models just compare two images giving measure of their similarity [6], or only analyze the presence or not of texture without given a measure value [7].

In this paper we face the problem of relating the texture measures with the human perception of texture. Concretely, we focus our study in the coarsenessfineness property, proposing models which relate representative measures of texture (usually some statistic) with its human perception of fineness. For this purpose, a wide variety of measures are analyzed, and human assessments about texture perception are collected. From this data, a functional relationship between the measures and the human assessments is learnt.

The rest of the paper is organized as follows. In section 2 we introduce our methodology to obtain the functions that model the fineness textural property. In section 3 we show the results of applying the models and the main conclusions and future work are sumarized in section 4 .

\section{Perceptually-Based Fineness Functions}

Focusing our study on fineness-coarseness property, there are different measures over the literature that, given an image, capture the fineness (or coarseness) presence in the sense that the greater the value given by the measure, the greater the perception of texture. However two main drawbacks are found: on the one hand, there is no perceptual relationship between that value and the degree that humans perceive the texture; on the other hand, there are no thresholds that, given a certain measure, allow to decide whether there is fine texture, coarse texture or something intermediate (i.e. there are no intervals on the measure domain allowing for textural interpretation).

In this paper, we face the above questions by proposing a function of fineness perception defined on the domain of a given subset of fineness and coarseness measures. Let $\mathcal{P}=\left\{P_{1}, \ldots, P_{K}\right\}$ be the set of $K$ fineness measures analyzed in this paper (showed in the first column of Table 1), and let $\mathcal{F}=\left\{F_{1}, \ldots, F_{K^{\prime}}\right\} \subseteq$ $\mathcal{P}$ be a subset of $K^{\prime} \leq K$ measures selected from $\mathcal{P}$. Thus, we propose to model the fineness perception by means of a function $\mathcal{T}_{\mathcal{F}}$ defined a: 1

$$
\mathcal{T}_{\mathcal{F}}: \mathbb{R}^{K^{\prime}} \rightarrow[0,1]
$$

with $K^{\prime}$ being the cardinality of $\mathcal{F}$. The value given by the function $\mathcal{T}_{\mathcal{F}}$ will indicate how fine or coarse is the texture present in an image. Thus, a value of 1 will mean fineness presence while a value of 0 will mean no fineness presence (i.e. coarseness presence). Therefore, values between 0 and 1 will represent the degree of how fine the image is perceived (the closer the value to 1 , the greater the perception of fineness).

In this paper, we propose to obtain $\mathcal{T}_{\mathcal{F}}$ by fitting a suitable function relating fineness measures with the degree of fineness perceived by humans. To learn this

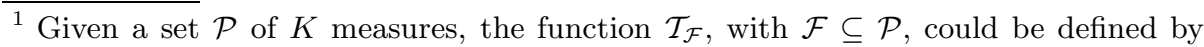
using all the measures $\left(K^{\prime}=K\right)$, or a subset of them $\left(K^{\prime}<K\right)$. 

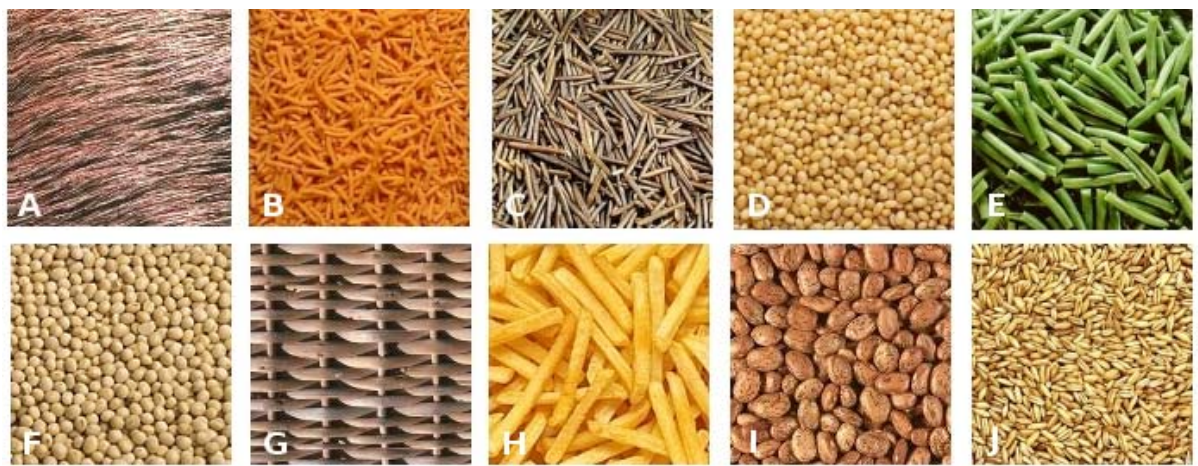

Fig. 1. Some examples of images with different degrees of fineness

relationship, a set $\mathcal{I}=\left\{I_{1}, \ldots, I_{N}\right\}$ of $N$ images covering different degrees of fineness will be used. For each image $I_{i} \in \mathcal{I}$, we will have (a) a set of fineness values obtained by applying the measures $\mathcal{F}$ to the image $I_{i}$ and (b) assessments about of the perception of fineness in the image $I_{i}$ obtained from subjects. To get these assessments, a poll will be performed (section 2.1). Therefore, for each image, we will have pairs of measure-assessment values, so a fitting will be applied in order to obtain a suitable function (section 2.2).

\subsection{Assessment Collection}

In this section, the way to obtain the fineness assessments from the image set $\mathcal{I}$ will be described. From now on, we will note as $\Gamma=\left\{v^{1}, \ldots, v^{N}\right\}$ the set of fineness assessment values associated to $\mathcal{I}$, with $v^{i}$ being the aggregated value representing the degree of fineness perceived by humans in the image $I_{i} \in \mathcal{I}$.

The texture image set. A set $\mathcal{I}=\left\{I_{1}, \ldots, I_{N}\right\}$ of $N=80$ images representative of the fineness-coarseness property has been selected. Figure 1 shows some images extracted from the set $\mathcal{I}$. The selection was done satisfying the following properties: (1) it covers the different presence degrees of fineness-coarseness, (2) the number of images for each degree is representative enough, and (3) each image shows, as far as possible, just one degree of fineness. Due to the third property, each image can viewed as "homogeneous" respect to the fineness degree represented, i.e., if we select two random windows (with a dimension which does not "break" the original texture primitives and structure), the perceived fineness will be the same for each window (and also respect to the original image).

As we explained, given an image $I_{i} \in \mathcal{I}$, a set of measures $\mathcal{F}$ will be applied on it. In fact, and thanks to the third property, we really can apply these measures for each subimage, assuming that the human assessment associated to that subimage will be the human assessment associated to the whole image. 
From now on, we will note as $\mathbf{M}_{w}^{i}=\left[m_{1}^{i, w}, \ldots, m_{K^{\prime}}^{i, w}\right]$ the vector of measures for the $w$-th window of the image $I_{i}$, with $m_{k}^{i, w}$ being the result of applying the measure $F_{k} \in \mathcal{F}$ to the $w$-th window of the image $I_{i}$. Therefore, and using the previous notation, the set of valid pairs used for the fitting procedure will be given by $\Psi_{F}=\left\{\left(\mathbf{M}_{w}^{i}, v^{i}\right), i=1, \ldots, N ; w=1, \ldots, W\right\}$, with $N$ being the number of images and $W$ the number of windows considered for each image.

The poll. Given the image set $\mathcal{I}$, the next step is to obtain assessments about the perception of fineness from a set of subjects. From now on, we shall denote $\Theta^{i}=\left[o_{1}^{i}, \ldots, o_{L}^{i}\right]$ the vector of assessments obtained from $L$ subjects for image $I_{i}$. To get $\Theta^{i}$, subjects will be asked to assign images to classes, so that each class has associated a perception degree of texture. In particular, 20 subjects have participated in the poll and 9 classes have been considered. The first nine images in figure 1 show the nine representative images for each class used in this poll. It should be noticed that the images are decreasingly ordered according to the perception degree of fineness.

Assessment aggregation. Our aim at this point is to obtain, for each image $I_{i} \in \mathcal{I}$, one assessment $v^{i}$ that summarizes the vector of assessments $\Theta^{i}$ given by the different subjects about the perception degree of fineness. To aggregate opinions we have used an OWA operator guided by the quantifier "the most" 8] which allows to represent the opinion of majority of the polled subjects.

\subsection{Fitting the Function}

At this point, the aim is to obtain, for a given subset of measures $\mathcal{F} \subseteq \mathcal{P}$, the corresponding function $\mathcal{T}_{\mathcal{F}}$ by fitting a suitable curve to the set $\Psi_{\mathcal{F}}$. In this paper, the robust fitting based on M-estimators (a generalization of the least squares fitting)) has been used [15]. For each image $I_{i} \in \mathcal{I}, W=2000$ subimages of size $32 \times 32$ have been considered (so 16000 points have been used for the fitting).

For defining $\mathcal{F}$, we have focused our analysis on the cases of $K^{\prime}=1$ and $K^{\prime}=2$. The unidimensional case $\left(K^{\prime}=1\right)$ will allow to study each measure separately, comparing its goodness respect to the others. In the bidimensional case $\left(K^{\prime}=2\right)$, we will combine two measures in order to improve the individual ones (all the combinations of two elements have been considered). For higher dimensions $\left(K^{\prime} \geq 3\right)$, the fitting based on M-estimators is too complex, so new methods for finding $\mathcal{T}_{\mathcal{F}}$ need to be considered (for example, some kind of heuristic approaches). The case of $K^{\prime} \geq 3$ will be considered in future works.

Both the unidimensional and bidimensional fittings are analyzed in the next subsections. In both cases, the following considerations will be taked into account: (1) $\mathcal{T}_{F}$ should be a monotonic function, and (2) the values $\mathcal{T}_{\mathcal{F}}(x)=0$ and $\mathcal{T}_{\mathcal{F}}(x)=1$ should be achieved from a certain value. It should be noticed that there is an error related to the fitting, calculated as the mean of the absolute difference between the data points and the curve. This error can also be viewed as a goodness measurement of the measures used in $\mathcal{F}$. 
Table 1. Fitting errors related to unidimensional and bidimensional fitting and parameter values for the measures with least error

\begin{tabular}{|c|c|c|c|c|}
\hline \multicolumn{2}{|l|}{ Unidimensional fitting } & \multicolumn{3}{|c|}{ Bidimensional fitting } \\
\hline Measure & Error & Measure x & Measure y & Error \\
\hline Correlation 3 & 0.1466 & FD & Amadasun & 0.1095 \\
\hline Amadasun 1 & 0.1515 & Correlation & ED & 0.1332 \\
\hline Abbadeni 4 & 0.1880 & Correlation & Tamura & 0.1339 \\
\hline Fractal dim. (FD) 9 & 0.1891 & Amadasun & Correlation & 0.1354 \\
\hline Tamura 2] & 0.1994 & Abbadeni & Tamura & 0.1373 \\
\hline Edge density (ED) [3] & 0.2044 & Amadasun & ED & 0.1431 \\
\hline DGD 10 & 0.2120 & Correlation & Abbadeni & 0.1433 \\
\hline Local Homogeneity 3 & 0.2156 & Abbadeni & Amadasun & 0.1444 \\
\hline Short Run Emphasis 11 & 0.2211 & Correlation & FD & 0.1455 \\
\hline SNE 12 & 0.2365 & Abbadeni & FD & 0.1475 \\
\hline Weszka [5] & 0.2383 & Tamura & Amadasun & 0.1529 \\
\hline Newsam [13, Entropy 3 , & WS & FD & Tamura & 0.1634 \\
\hline Uniformity 3, FMPS 14 & WSD & ED & Tamura & 0.1636 \\
\hline Variance 3 & & $\mathrm{ED}$ & FD & 0.1764 \\
\hline Contrast 3] & $\mathrm{NR}$ & Abbadeni & ED & 0.1799 \\
\hline \multicolumn{2}{|c|}{$\begin{array}{l}\text { Parameters for Correlation: } \\
a_{3}=-1.8383, a_{2}=1.2338, a_{1}=-1.2079 \\
a_{0}=1.0339, \alpha=0.7685, \beta=0.0289\end{array}$} & \multicolumn{3}{|c|}{$\begin{array}{l}\text { Parameters for }\{F D, \text { Amadasun }\}: \\
a_{9}=1.1169, a_{8}=-7.60 \mathrm{E}-4, a_{7}=0, a_{6}=-8.53 \mathrm{E}-3, a_{5}=-11.388 \\
a_{4}=6.17 \mathrm{E}-2, a_{3}=0.2289, a_{2}=36.81, a_{1}=-1.2616, a_{0}=-34.76\end{array}$} \\
\hline
\end{tabular}

Unidimensional fitting. Regarding the above properties, in the case of $K^{\prime}=1$ we propose to define $\mathcal{T}_{\mathcal{F}}$ as a function of the form 2

$$
\mathcal{T}_{\mathcal{F}}\left(x ; a_{n} \ldots a_{0}, \alpha, \beta\right)= \begin{cases}0 & x<\alpha \\ \operatorname{poly1}\left(x ; a_{n} \ldots a_{0}\right) & \alpha \leq x \leq \beta, \\ 1 & x>\beta\end{cases}
$$

with $\operatorname{poly} 1\left(x ; a_{n} \ldots a_{0}\right)$ being a polynomial function

$$
\operatorname{poly} 1\left(x ; a_{n} \ldots a_{0}\right)=a_{n} x^{n}+\ldots+a_{1} x^{1}+a_{0}
$$

In our proposal, the parameters $a_{n} \ldots a_{0}, \alpha$ and $\beta$ of the function $\mathcal{T}_{\mathcal{F}}$ are calculated by carrying out the robust fitting on $\Psi_{\mathcal{F}}$. For the polynomial function, the cases of $\mathrm{n}=1,2,3$ have been considered.

Table 1 shows, for each measure $F_{k} \in \mathcal{F}$, the least error obtained in the fitting process (sorted in increasing order of the errors). The parameter values corresonding to the lowest error fitting (correlation) are also shown in Table 1 . It should be noticed that we haven't carried out the fitting with six of the measures. Four of them (marked with WSD) are rejected because their values are affected by the window size. The other two (marked with NR) produce a diffuse cloud of points $\Psi_{F}$ with no representative information.

\footnotetext{
${ }^{2}$ Note that this function is defined for measures that increase according to the perception of fineness. For those that decreases, the function needs to be changed appropriately.
} 
Bidimensional fitting. In the case of $K^{\prime}=2$, we define $\mathcal{T}_{\mathcal{F}}$ as a function of the form:

$$
\begin{gathered}
\mathcal{T}_{\mathcal{F}}\left(x, y ; a_{(n !+n)} \ldots a_{0}\right)=\mathcal{T}_{\mathcal{F}}(x, y ; \text { coef })= \\
= \begin{cases}0 & \text { poly } 2(x, y ; \text { coef })<0, \\
\operatorname{poly} 2(x, y ; \text { coef }) & 0<\operatorname{poly} 2(x, y ; \text { coef })<1, \\
1 & \text { poly } 2(x, y ; \text { coef })>1\end{cases}
\end{gathered}
$$

with poly2 $(x, y$; coef $)$ being a polynomial function of two variables

$$
\operatorname{poly} 2\left(x, y ; a_{(n !+n)} \ldots a_{0}\right)=\sum_{i=0}^{n} \sum_{j=0}^{i} a_{(i !+j)} x^{j} y^{i-j}
$$

As in the unidimensioanl case, the parameters $a_{(n !+n)} \ldots a_{0}$ of the function $\mathcal{T}_{\mathcal{F}}$ are calculated by carrying out the robust fitting on $\Psi_{\mathcal{F}}$. For the polynomial function, the cases of $n=1,2,3$ have been considered.

Table 1 shows the least fitting error obtained for each pair of measures (only the pairs compound by the first six measures in the unidimensional case are shown). It can be noticed that the use of bidimensional functions reduces the error and provides better fineness models than the unidimensional ones. In addition, the combination of two measures improves the results obtained for each measure separately. In our experiments, the pair $\mathcal{F}=\{F D$, Amadasun $\}$ gives the best results. The parameter values corresponding to this member function are shown in Table 1

\section{Results}

In this section, the function $\mathcal{T}_{\mathcal{F}}$ with the least fitting error, corresponding to the pair of measures $\mathcal{F}=\{F D$, Amadasun $\}$, will be applied in order to analyze the performance of the proposed model.

Let's consider Figure 2(A) corresponding to a mosaic made by several images, each one with a different increasing perception degree of fineness. The fineness of each subimage has been calculated using the proposed model and the results are shown in Figure 2(B), being $v$ the value of the human assessment and $f$ the value computed by our model. It can be noticed that our model captures the evolution of the perception degrees of fineness.

Figure 2(C) shows a mapping from the original image to its fineness values using the proposed model. For each pixel in the original image, a centered window of size $32 \times 32$ has been analyzed and its fineness has been calculated. The histogram of this image is shown below, where we can see clearly four differentiated peaks, corresponding to the four different fineness degrees in the image. Figures 2(D) and 2(E) show a mapping from the original image using the Amadasun and FD measures directly. It can be seen that these mappings don't provide as much perceptual information as Figure 2(C). Furthermore, their histograms show that the four peaks can't be obtained with the measures separately. 

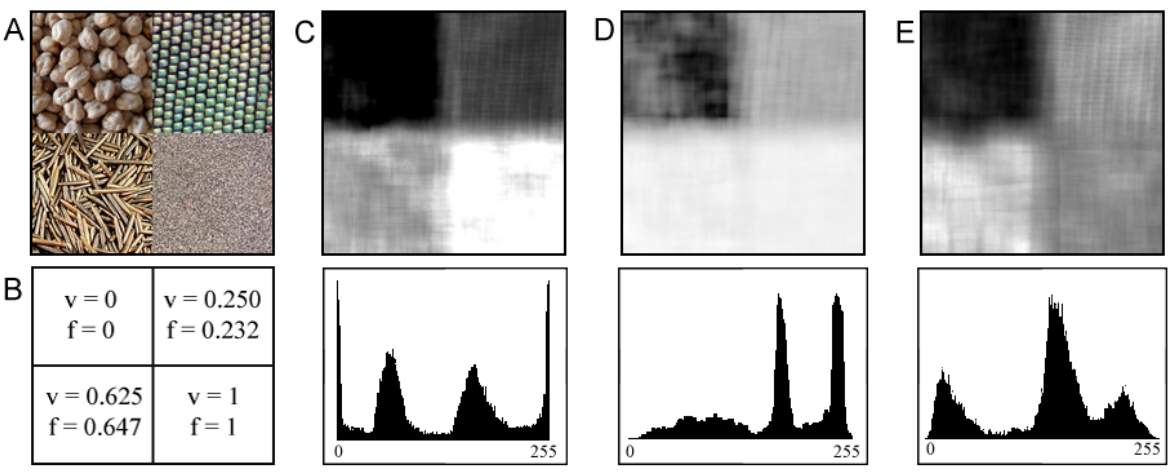

Fig. 2. Results for a mosaic image. (A) Original mosaic image (B) Fineness values obtained with the proposed model for $\mathcal{F}=\{F D$, Amadasun $\}$, with $v$ being the value of the human assessment and $f$ the value computed by our model (C) Mapping from the original image to its fineness values using the proposed model (D)(E) Mapping using the individual measures Amadasun and FD, respectively.

Figure 3 presents an example where the proposed model has been employed for pattern recognition. Concretely, it shows a microscopy image (Figure 3(A)) corresponding to the microstructure of a metal sample. The lamellae indicates islands of eutectic, which need to be separated from the uniform light regions. The brightness values in regions are similar to the background ones, so texture information is needed for extracting the uniform areas. This fact is showed in Figure 3(B1,B2), where a thersholding on the original image is displayed (homogeneous regions cannot be separated from the textured ones).

Figure 3(C1) shows a mapping from the original image to its fineness values using the proposed model (a window of size $20 \times 20$ has been used). It can be noticed that uniform regions correspond to areas with low values of fineness (i.e., high coarseness), so if only the pixels with fineness values lower than 0.1 are selected (which it is equivalent to a coarseness degree upper than 0.9), the uniform light regions emerge with ease (Figure 3)(C2,C3)).
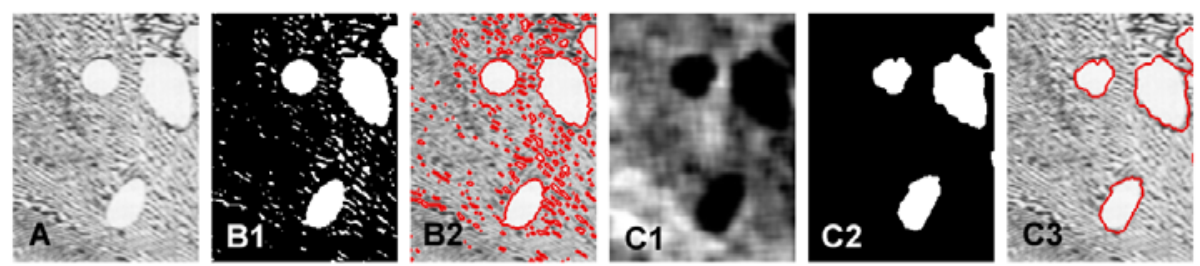

Fig. 3. (A) Original image (B1) Binary image obtained by thresholding the original image (B2) Region outlines of B1 superimposed on original image (C1) Fineness mapping obtained with our model from the original image (C2) Binary image obtained by thresholding C1 (C3) Region outlines of C2 superimposed on original image 


\section{Conclusions}

In this paper, functions relating computational fineness measures with the human perception of this type of texture property have been proposed. In order to obtain assessments about fineness perception, a group of human subjects have been polled. From the collected data, a robust fitting procedure has been applied, resulting unidimensional and bidimensional functions with perceptual meaningful. In our study, the combination of the Amadasum and the FD measures has given the best fitting. The results show a high performance of the model respect to the assessments given by subjects.

\section{References}

1. Amadasun, M., King, R.: Textural features corresponding to textural properties. IEEE Transactions on Systems, Man and Cybernetics 19(5), 1264-1274 (1989)

2. Tamura, H., Mori, S., Yamawaki, T.: Textural features corresponding to visual perception. IEEE Trans. on Systems, Man and Cybernetics 8, 460-473 (1978)

3. Haralick, R.: Statistical and structural approaches to texture. Proceedings IEEE 67(5), 786-804 (1979)

4. Abbadeni, N., Ziou, N., Wang, D.: Autocovariance-based perceptual textural features corresponding to human visual perception. In: Proc. of 15th International Conference on Pattern Recognition, vol. 3, pp. 901-904 (2000)

5. Wezska, J., Dyer, C., Rosenfeld, A.: A comparative study of texture measures for terrain classification. IEEE Trans. on SMC 6, 269-285 (1976)

6. Fahmy, G., Black, J., Panchanathan, S.: Texture characterization for joint compression and classification based on human perception in the wavelet domain. IEEE Transactions on Image Processing (2006)

7. Chen, J., Pappas, T., Mojsilovic, A., Rogowitz, B.: Adaptive perceptual colortexture image segmentation. IEEE Transactions on Image Processing (2005)

8. Yager, R.: On ordered weighted averaging aggregation operators in multicriteria decisionmaking. IEEE Trans. on SMC 18(1), 183-190 (1988)

9. Peleg, S., Naor, J., Hartley, R., Avnir, D.: Multiple resolution texture analysis and classification. IEEE Trans. on PAMI (4), 518-523 (1984)

10. Kim, S., Choi, K., Lee, D.: Texture classification using run difference matrix. In: Proc. of IEEE 1991 Ultrasonics Symposium, vol. 2, pp. 1097-1100 (1991)

11. Galloway, M.: Texture analysis using gray level run lengths. Computer Graphics and Image Processing 4, 172-179 (1975)

12. Sun, C., Wee, W.: Neighboring gray level dependence matrix for texture classification. Computer Vision, Graphics and Image Processing 23, 341-352 (1983)

13. Newsam, S., Kammath, C.: Retrieval using texture features in high resolution multi-spectral satellite imagery. In: Data Mining and Knowledge Discovery: Theory, Tools, and Technology VI, SPIE Defense and Security (2004)

14. Yoshida, H., Casalino, D., Keserci, B., Coskun, A., Ozturk, O., Savranlar, A.: Wavelet-packet-based texture analysis for differentiation between benign and malignant liver tumours in ultrasound images. Phy.in Med. Bio. 48, 3735-3753 (2003)

15. Beaton, A., Tukey, J.: The fitting of power series, meaning polynomials, illustrated on band-spectroscopic data. Technometrics 16, 147-185 (1974) 